\title{
ON COURNOT-NASH EQUILIBRIUM DISTRIBUTIONS FOR GAMES WITH A NONMETRIZABLE ACTION SPACE AND UPPER SEMICONTINUOUS PAYOFFS
}

\author{
M. ALI KHAN
}

\begin{abstract}
We report results on the existence of a Cournot-Nash equilibrium distribution for games in which the action space is not necessarily metrizable and separable and the payoff functions are not necessarily continuous. Our work relies on the theory of Radon measures as developed by Schwartz-Topsoe and on the epitopology as developed by Dolecki-Salinetti-Wets
\end{abstract}

\section{INTRODUCTION}

Mas-Colell [16] presents a reformulated version of Schmeidler's [21] results on the existence of Cournot-Nash equilibria in games with a continuum of players. Taking his lead from Hart-Hildenbrand-Kohlberg [9], Mas-Collell views a game as a probability measure $\mu$ on the space of payoff functions $\mathscr{U}_{A}$, each such function being defined on the product of the given strategy or action space $A$ and the space of probability measures on $A, \mathscr{M}$. The dependence of a player's payoff on the actions of the other players is summarized by the dependence of the payoff on $\mathscr{M}$. A Cournot-Nash equilibrium is then formulated as a measure $\tau$ on the product space $A \times \mathscr{U}_{A}$ such that (i) the marginal of $\tau$ on $\mathscr{U}_{A}$ is the game $\mu$ itself, and (ii) the maximizing action payoff pairs, given $\tau$, have full $\tau$ measure. Under the assumption of $A$ being a compact metric space and the payoff functions being continuous, Mas-Colell shows the existence of a Cournot-Nash equilibrium as a consequence of the Ky Fan fixed-point theorem and with the space of measures being endowed with the weak ${ }^{*}$ topology.

The hypothesis of a compact action space is natural enough but the assumption of it being metric is less easily justified. Indeed, in the context of a Banach space which is not necessarily separable, weakly compact action spaces are not necessarily metrizable. The same is true for weak ${ }^{*}$ compact sets of Banach spaces whose preduals are not separable. It is thus natural to ask whether MasColell's theorem can be proved without the metric hypothesis. This is the first question we investigate in this note.

Received by the editors March 2, 1988.

1980 Mathematics Subject Classification (1985 Revision). Primary 28C15, 90D40, 90A14.

Key words and phrases. Cournot-Nash equilibrium distribution, compact Hausdorff action space, Radon measure, disintegration, compact-open topology, topology of epiconvergence. 
An answer to this question is of interest not only because it examines the robustness of Mas-Colell's conception but also because it leads naturally to the study of measures on a product space, one of whose elements is compact but not metric and the other is metrizable but not separable. In particular, $A$ not being separable leads to $\mathscr{U}_{A}$ not being separable (see, for example, Willard [25, p. 282]). Furthermore, it is far from clear how one could accommodate nonseparable action spaces in the theory as originally laid out by Schmeidler and developed in Khan [13] and Khan-Papageorgiou [14, 15]. This theory relies crucially on measurable selection theorems.

In the context of our first question, we present three results. First, we show that the metric assumption on $A$ can be dispensed with in Mas-Colell's theorem if a game is defined as a Radon (tight) measure on the space of payoff functions. Since every abstract measure on a complete, separable metric space is a Radon measure, our result is a generalization of that of Mas-Colell. Indeed, it is precisely the loss of the validity of this observation in the nonmetrizable setup that leads to complications of the argument. For example, Theorem 5.8 in Parthasarathy [19, Chapter II] on the existence of a Radon measure is no longer available; nor is Theorem 3.2 in Billingsley [3], which characterizes weak convergence of a measure in terms of the weak convergence of its marginals (also see Parthasarathy [19, Chapter III, Lemma 1.1]). Nevertheless, one can appeal to results in Schwartz [22] and Topsoe [23] and provide a proof along the lines laid by Mas-Colell. These results also allow us to make an observation regarding the closedness of the graph of the Cournot-Nash equilibrium correspondence. We present sufficient conditions under which this can be strengthened to upper hemicontinuity. This constitutes the second result of the paper.

Mas-Colell also asked if there exist a Cournot-Nash equilibrium distribution $\tau$ and a measurable function $f$ from the space of payoffs to the space of actions such that $\tau$ gives full measure to the graph of $f$. He called such equilibria symmetric and showed their existence for finite actions, atomless games. In an analogous vein, we investigate Cournot-Nash eqilibrium distributions which can be viewed as a suitable integral of a measurable function from the space of payoffs to measures on the action space or from the action space to measures on the space of payoffs. We call these disintegrated Cournot-Nash equilibria and establish their existence as a consequence of a recent result of Edgar [8].

Since every upper semicontinuous function attains its maximum on a compact set, it is natural to ask if the above theory genralizes to upper semicontinuous, rather than continuous, payoffs. This is the second question we investigate in this note. An answer to this question is of particular interest in light of recent emphasis on games with discontinuous payoffs; see, for example, Dasgupta-Maskin [5] and their references.

The principal technical difficulty with such an extension lies in the fact that the sup norm topology is no longer available and one needs to formulate a topology on the space of payoffs that is able to fulfill the demands made on it in the course of the proofs. Such a topology is available from the recent 
work of Dolecki-Salinetti-Wets [6] and is simply motivated by the observation that every lower semicontinuous function has a closed epigraph. There are, of course, several topologies on the space of closed subsets of a topological space, and Dolecki et al. [6] choose the topology of closed convergence. This topology is, by now, well understood in mathematical economics; see, for example, Hildenbrand [10].

It needs to be underscored, however, that our extension of the theory to upper semicontinuous payoffs involves a variant of Mas-Colell's formulation of a game. Although we believe this variant to have independent interest, it would be nice to know if upper semicontinuous payoffs could be handled in the original formulation of Mas-Colell. We doubt this to be possible but have no counterexamples. On the technical side, it may be useful to point out that even though the theory with continuous payoffs provides the essential pointers, our extension to upper semicontinuous payoffs makes considerable demands on the properties of the compact-open topology.

The plan of the paper is as follows. $\S 2$ presents some preliminary results on the space of Radon measures on a compact Hausdorff space. These results are well known but are not found in standard texts such as Billingsley [3], Hildenbrand [10], or Parthasarathy [19]. $\S 3$ develops the theory with continuous payoffs and $\S 4$ with upper semicontinuous ones. $\S 5$ discusses the interrelationship between the two formulations pursued in $\S \S 3$ and 4 and presents some open questions. $\S 6$ is devoted to the proofs.

\section{MATHEMATICAL PRELIMINARIES}

In this section we collect some results on the space of Radon probability measures on topological spaces that are not necessarily separable or metrizable.

Let $X$ be a Hausdorff topological space and $\mathscr{M}_{+}(X)\left(\overline{\mathscr{M}}_{+}(X)\right)$ the space of all nonnegative, finite measures defined on $\mathscr{B}(X)(\mathscr{\mathscr { B }}(X))$, the Borel $\sigma$-algebra (Baire $\sigma$-algebra) on $X, \mu \in \mathscr{M}_{+}(X)$ is said to be a Radon measure or tight if

$$
\mu(B)=\operatorname{Sup}\{\mu(K): K \text { compact, } K \subset B\} \text { for all } B \in \mathscr{B}(X) .
$$

We shall denote the set of all Radon measures on $(X, \mathscr{B}(X))$ by $\mathscr{M}_{+}(X, t)$ and the set of all probability measures and Radon probability measures by $\mathscr{M}_{+}^{1}(X)$ and $\mathscr{M}_{+}^{1}(X, t)$, respectively. $\overline{\mathscr{M}}_{+}^{1}(X)$ will denote the subset of probability measures in $\overline{\mathscr{M}}_{+}(X)$.

Theorem 2.1. If $A \in \mathscr{B}(X)$ and $\mu \in \mathscr{M}_{+}(X, t)$, then $\mu \mid A$, the restriction of $\mu$ to $A$, is a measure in $\mathscr{M}_{+}(A, t)$.

Proof. See Topsoe [23, Preliminaries, p. 14].

Following Topsoe [23], Schwartz [22], and others, we define the weak topology on $\mathscr{M}_{+}(X)$ as the weakest topology on $\mathscr{M}_{+}(X)$ for which every map $\mu \rightarrow \mu(f)$, where $f: X \rightarrow R$ is bounded and upper semicontinous, is upper 
semicontinuous. Note that

$$
\mu(f)=\int_{X} f(x) d \mu(x)
$$

The induced topology on $\mathscr{M}_{+}(X, t)$ and $\mathscr{M}_{+}^{1}(X, t)$ will also be called the weak topology and these spaces are always assumed to be endowed with this topology.

Theorem 2.2. $\mathscr{M}_{+}(X, t)$ is Hausdorff for any Hausdorff topological space $X$.

Proof. See Topsoe [23, Theorem 11.2] or Schwartz [22, Proposition 2, p. 371].

Theorem 2.3. $\mathscr{M}_{+}^{1}(X, t)$ is compact if and only if $X$ is compact.

Proof. See, for example, the notes to $\S 11$ in Topsoe [23, p. 76]. Also see Schwartz [22, p. 379].

Theorem 2.4. Let $\left\{\gamma^{\alpha}\right\}$ be a net in $\mathscr{M}_{+}(X)$ and $\gamma$ an element of $\mathscr{M}_{+}(X)$. Then $\gamma^{\alpha}$ converges to $\gamma$ if and only if

(i) $\lim \sup \gamma^{\alpha}(F) \leq \gamma(F)$ for all closed subsets $F$ of $X$,

(ii) $\lim \sup \gamma^{\alpha}(X)=\gamma(X)$

Proof. See Theorem 8.1 of Topsoe [23, p. 40].

Our next two results relate convergence of a net of measures to convergence of their marginals. For a measure $\rho$ on a product space $S \times T$, let $\rho_{S}, \rho_{T}$ be the marginal measures on $S$ and $T$, respectively.

Theorem 2.5. Let $\left\{\gamma^{\alpha}\right\}$ be a net in $\mathscr{M}_{+}^{1}(S \times T, t)$ which converges to $\gamma$ in $\mathscr{M}_{+}^{1}(S \times T, t)$. Then $\gamma_{i}^{\alpha}$ converges to $\gamma_{i}, i=S, T$.

Proof. Without loss of generality, let $i=S$ and pick an arbitrary closed subset $F$ of $S$. Then $(F \times T)$ is closed and we appear to Theorem 2.4 to finish the proof.

Theorem 2.6. Let $S$ and $T$ be completely regular spaces and $\left\{\gamma^{\alpha}\right\}$ be a net in $\mathscr{M}_{+}(S \times T, t)$. If

$$
\gamma_{S}^{\alpha} \rightarrow \mu, \quad \gamma_{T}^{\alpha} \rightarrow \nu
$$

for some $\mu \in \mathscr{M}_{+}(S, t)$ and some $\nu \in \mathscr{M}_{+}(T, t)$, then $\left\{\gamma^{\alpha}\right\}$ has a limit point $\gamma \in \mathscr{M}_{+}(S \times T, t)$ with marginals $\mu$ and $\nu$.

Proof. See the proof of Lemma 5.1 in Hoffman-Jorgenson [11].

Our next result shows that Borel probability measures with finite support are dense in $\mathscr{M}_{+}^{1}(X)$.

Theorem 2.7. Let $\mathscr{E}$ be the set of Dirac point measures on $(X, \mathscr{B}(X))$. Then the closed convex hull of $\mathscr{E}$ is $\mathscr{M}_{+}^{1}(X)$.

Proof. See the proof of Theorem 11.1 in Topsoe [23, p. 48].

Next, we present a general result on the disintegration of measures. For any two measurable spaces $(F, \mathscr{F}),(G, \mathscr{G})$, we shall denote the product $\sigma$-algebra on $F \times G$ by $\mathscr{F} \times \mathscr{G}$. Furthermore, for any $W \in \mathscr{F} \times \mathscr{G}$, and any $f \in F$, let 
$W_{f}=\{g \in G:(f, g) \in W\}$. The sections of $W$ on $G$ are defined similarly. Also recall from Edgar [8] that $\mu \in \overline{\mathscr{M}}_{+}^{1}(X)$ is said to be Radon or tight if and only if for all $\varepsilon>0$ there exists a compact set $K \subset X$ such that for every $B \in \overline{\mathscr{B}}(X), K \subset B, \mu(B) \geq 1-\varepsilon$.

Theorem 2.8. Let $X$ be a Hausdorff topological space, $(S, \mathscr{S})$ a measurable space, $\tau$ a probability measure on $(S \times X), \mathscr{S} \times \overline{\mathscr{B}}(X))$, and the marginal of $\tau$ on $X, \tau_{X}$, Radon. Then there exists a family of probability measures on $X$, $\left(\rho_{s}\right)_{s \in S}, \rho_{s} \in \overline{\mathscr{M}}_{+}^{1}(X)$, such that for any $W \in \mathscr{S} \times \overline{\mathscr{B}}(X)$,

(i) $h_{W}: S \rightarrow R, h_{W}(s)=\rho_{s}\left(W_{s}\right)$, is measurable;

(ii) $\tau(W)=\int_{S} h_{W}(s) d \tau_{S}(s)$.

Proof. See the proof of Theorem 3.1 in Edgar [8].

Finally, for the record, we state

Theorem 2.9. Let $\mu$ be a Borel probability measure on a topological space $(X, \tau)$. If $\tau$ is metrizable with a complete metric and is separable, then $\mu \in$ $\mathscr{M}_{+}^{1}(X, t)$.

Proof. See Billingsley [3, Theorem 1.4] or Parthasarathy [19, II, Theorem 3.2]. More generally, see Topsoe [23, p. 16].

\section{The MOdel AND Results: Continuous PAYOFFS}

Let $A$ be a nonempty, compact Hausdorff space of actions. A player is characterized by a continuous utility function $u: A \times \mathscr{M}_{+}^{1}(A, t) \rightarrow R$. Let $\mathscr{U}_{A}$ be the space of continuous utility functions endowed with the supremum norm topology; recall that $\mathscr{M}_{+}^{1}(A, t)$ is compact by virtue of Theorem 2.2. We can now state

Definition 3.1. A game is a Borel probability measure $\mu$ on $\mathscr{U}_{A}$.

Definition 3.2. A Borel probability measure $\tau$ on $\mathscr{U}_{A} \times A$ is a Cournot-Nash equilibrium distribution of a game $\mu$ if

(i) $\tau_{\mathscr{U}_{A}}=\mu$;

(ii) $\tau\left(\left\{(u, a): u\left(a, \tau_{A}\right) \geq u\left(A, \tau_{A}\right)\right\}\right) \equiv \tau\left(B_{\tau}\right)=1$.

We can now present our results.

Theorem 3.1. If $\mu \in \mathscr{M}_{+}^{1}\left(\mathscr{U}_{A}, t\right)$, there exists a Cournot-Nash equilibrium distribution for the game $\mu$.

Remark. The proof shows that the Cournot-Nash equilibrium distribution is Radon, i.e., an element of $\mathscr{M}_{+}^{1}\left(A \times \mathscr{U}_{A}, t\right)$.

Corollary (Mas-Colell). If $A$ is metrizable, then the theorem is true for any Borel probability measure on $\mathscr{U}_{A}$.

We also present a result on the upper hemicontinuity (upper semicontinuity in the terminology of Berge [2]) of the Cournot-Nash correspondence. For any game $\mu$, let $\Gamma(\mu)$ denote its set of Cournot-Nash equilibria. 
Theorem 3.2. $\Gamma: \mathscr{M}_{+}^{1}\left(\mathscr{U}_{A}, t\right) \rightarrow \mathscr{M}_{+}^{1}\left(A \times \mathscr{U}_{A}, t\right)$ has a closed graph.

For any compact set $S$ in $R$, let $\mathscr{U}_{A}^{S}$ be the subspace of $\mathscr{U}_{A}$ consisting of continuous functions from $A \times \mathscr{M}_{+}^{1}(A, t)$ into $S$. Let $\mathscr{J}$ be a closed, equicontinuous subset of $\mathscr{U}_{A}^{S}$; see, for example, Nagata [18, p. 279] for a definition of equicontinuity. We can now appeal to the Ascoli-Arzela theorem and strengthen the conclusion of Theorem 3.2 to

Theorem 3.3. $\Gamma: \mathscr{M}_{+}^{1}(\mathscr{J}, t) \rightarrow \mathscr{M}_{+}^{1}(A \times \mathscr{J}, t)$ is an upper hemicontinuous mapping.

The interpretation of Theorem 3.3 is essentially the observation that "small" perturbations in the game lead to "small" perturbations in their Cournot-Nash equilibria if we limit ourselves to players that are "similar" in the sense of having equicontinuous payoffs.

Our final result requires a definition.

Definition 3.3. A Cournot-Nash equilibrium distribution $\tau$ can be disintegrated with respect to actions if there exists $\rho_{a} \in \overline{\mathscr{M}}_{+}^{1}\left(\mathscr{U}_{A}\right)$ such that for any $W \in$ $\mathscr{B}\left(\mathscr{U}_{A}\right) \times \overline{\mathscr{B}}(A)$,

(i) $h_{W}: A \rightarrow R, h_{W}(a)=\rho_{a}\left(W_{a}\right)$ is measurable;

(ii) $\tau(W)=\int_{A} \rho_{a}\left(W_{a}\right) d \tau_{A}(a)$.

$\tau$ can be disintegrated with respect to payoffs if there exist $\rho_{u} \in \overline{\mathscr{M}}_{+}^{1}(A)$ such that for any $W \in \mathscr{B}\left(\mathscr{U}_{A}\right) \times \overline{\mathscr{B}}(A)$,

(i) $g_{W}: \mathscr{U}_{A} \rightarrow R, g_{W}(u)=\rho_{u}\left(W_{u}\right)$ is measurable;

(ii) $\tau(W)=\int_{\mathscr{U}_{A}} \rho_{u}\left(W_{u}\right) d \mu(u)$.

Definition 3.4. A Cournot-Nash equilibrium distribution which can be disintegrated with respect to both actions and payoffs is said to be a disintegrated Cournot-Nash equilibrium distribution.

The interest in disintegrated distributions lies in the fact that they furnish a probability on the action space conditional on payoffs or, conditional on each action, a probability on the space of payoffs.

We can now present

Theorem 3.4. Every element of $\Gamma(\mu)$ can be disintegrated with respect to actions and if $\mu \in \mathscr{M}_{+}^{1}\left(\mathscr{U}_{A}, t\right)$, it can also be disintegrated with respect to payoffs. Hence, if $\mu \in \mathscr{M}_{+}^{1}\left(\mathscr{U}_{A}, t\right)$, there exists a disintegrated Cournot-Nash equilibrium distribution for the game $\mu$.

We finish this subsection by asking whether Theorems 3.1 to 3.3 are true for games which are not necessarily in $\mathscr{M}_{+}^{1}\left(\mathscr{U}_{A}, t\right)$ but are in $\mathscr{M}_{+}^{1}\left(\mathscr{U}_{A}\right)$, that is for games which are not necessarily Radon but are Borel probability measures. One may make two observations in this context. First, there certainly exist Borel probability measures even on compact Hausdorff spaces that are not Radon. 
The reader can see, for example, Dieudonné's construction [22, p. 45]. Second, and on the other hand, Edgar [8, p. 448] makes the observation that "it is consistent with the usual axioms of set theory (indeed, it follows from the Axiom of Constructibility) that if $X$ is a complete metric space, then every element of $\mathscr{M}_{+}(X)$ belongs to $\mathscr{M}_{+}^{1}(X, t)$, i.e., every Borel probability measure is tight." The reader need only be reminded that $\mathscr{U}_{A}$ is a complete metric space (see, for example, Willard [25, Theorem 42.10]).

\section{THE MODEL AND RESUlTS: UPPER SEMICONTINUOUS PAYOFFS}

In the previous section, a player was characterized by a continuous utility function from $A \times \mathscr{M}_{+}^{1}(A, t)$ to $R$. Such a function of two variables could be viewed alternatively as a family of functions from $A$ to $R$ that is parametrized by elements of $\mathscr{M}_{+}^{1}(A, t)$. Indeed, we can state the following elementary but important result; the reader is referred to Dugundji [7, Chapter 13] or Willard [25, Chapter 10] for a discussion of the compact-open topology.

Theorem 4.1. $u: A \times \mathscr{M}_{+}^{1}(A, t) \rightarrow R$ is a continuous function if and only if $\hat{u}: \mathscr{K}_{+}^{1}(A, t) \rightarrow C(A, R)$ is a continuous function with $C(A, R)$, the space of continuous functions from $A$ to $R$, endowed with the compact-open topology and where $[\hat{u}(\tau)](a)=u(a, \tau)$ for all $a \in A$ and $\tau \in \mathscr{M}_{+}^{1}(A, t)$.

In this section we exploit this alternative point of view and consider payoff functions that are only upper semicontinuous functions on the strategy sets but are continuous in the distributions on $A$ when viewed as a family of functions and endowed with a suitable topology. This space of continuous functions is further endowed with the compact-open topology and we are then in a position to define all the concepts of $\S 3$. A formalization of these ideas is warranted.

We continue to assume that $A$ is a nonempty, compact Hausdorff space of actions and consider upper semicontinuous utility functions $u: A \rightarrow \bar{R}$, where $\bar{R}$ is the space of extended reals. Following Dolecki-Salinetti-Wets (1983), we endow the space of upper semicontinuous utility functions with the hypotopology and denote the resulting space by $\mathscr{S}_{A}^{h}$. Two elements of $\mathscr{S}_{A}^{h}$ are "close" in the hypotopology if their hypographs are "close" in the topology of closed convergence. Recall that the hypograph of a function $f: X \rightarrow \bar{R}$ is given by the set $\{(x, \eta) \in X \times R: f(x) \geq \eta\}$. Since a function is upper semicontinuous if and only if its hypograph is a closed set, we have a well-defined topology. We now record the following result on the space $\mathscr{S}_{A}^{h}$, which is fundamental for this section.

Theorem 4.2. $\mathscr{S}_{A}^{h}$ is Hausdorff compact.

A player is characterized by a continuous function from $\mathscr{M}_{+}^{1}(A, t)$ to $\mathscr{S}_{A}^{h}$. Let $C\left(\mathscr{K}_{+}^{1}(A, t), \mathscr{S}_{A}^{h}\right)$ be the space of such functions endowed with the compact-open topology. For economy of notation, denote this space by $C\left(\mathscr{S}_{A}^{h}\right)$. We can define a game and a Cournot-Nash equilibrium distribution of a game 
by substituting $C\left(\mathscr{S}_{A}^{h}\right)$ for $\mathscr{U}_{A}$ in Definitions 3.1 and 3.2. We can now state the principal result of this section.

Theorem 4.3. Theorems $3.1,3.2$, and 3.4 are valid with $C\left(\mathscr{S}_{A}^{h}\right)$ substituted for $\mathscr{U}_{A}$. Moreover, Theorem 3.3 is valid with $\mathcal{J}$ an equicontinuous and closed subset of $C\left(\mathscr{S}_{A}^{h}\right)$.

Since $\mathscr{S}_{A}^{h}$ is Hausdorff compact by virtue of Theorem 4.1 , there exists a unique uniformity compatible with its topology (see, for example, Nagata [18, Theorem V1.15, p. 225]). This allows us to endow the set $C\left(\mathscr{M}_{+}^{1}(A, t), \mathscr{S}_{A}^{h}\right)$ with the topology of uniform convergence (see [18, p. 275] for definitions). For economy of notation, refer to this space as $C^{u}\left(\mathscr{S}_{A}^{h}\right)$. We can now state

Theorem 4.4. Theorem 4.3 is valid with $C^{u}\left(\mathscr{S}_{A}^{h}\right)$ substituted for $C\left(S_{A}^{h}\right)$.

Next we consider subspaces of $\mathscr{S}_{A}^{h}$. Consider first the subspace of upper semicontinuous functions from $A$ to $R$ instead of $\bar{R}$ and denote this by $\mathscr{S}_{A}^{h}$. Second, let $\mathscr{S}_{A}^{h}(b)$ denote the subspace of bounded upper semicontinuous functions from $A$ to $R . C\left(\mathscr{S}_{A}^{R}\right)$ and $C\left(\mathscr{S}_{A}^{h}(b)\right)$ will have the obvious meaning. We can now state

Theorem 4.5. Theorem 4.3 is valid with $C\left(\underline{\mathscr{S}}_{A}^{h}\right), C\left(\mathscr{S}_{A}^{h}(b)\right)$, or $C^{u}\left(\mathscr{S}_{A}^{h}(b)\right)$ substituted for $C\left(\mathscr{S}_{A}^{h}\right)$.

\section{SOME EXTENSIONS: CONTINUOUS PAYOFFS}

It hardly needs to be emphasized that the formulation of a player, and hence that of a game, pursued in $\S 3$ differs from that in $\S 4$. A natural question to ask is whether we could have incorporated upper semicontinuous payoffs in the formulation of $\S 3$. In other words, we could have characterized a player by an upper semicontinuous function from $A \times \mathscr{M}_{+}^{1}(A, t)$ to $\bar{R}$ and endowed the space of such functions with the hypotopology. To go a step further, we could have considered a subspace of functions continuous in $\mathscr{M}_{+}^{1}(A, t)$ and upper semicontinuous in $A$. We do not know if the results of $\S 3$ generalize to this setup and leave this as an open question.

Given this, we limit ourselves to continuous payoffs and ask what impact one formulation has on another. Specifically, this takes the form of the following two questions.

(i) What happens to the results in $\S 3$ when we endow the space of continuous payoffs with the topologies introduced in $\S 4$ ?

(ii) What happens to the results in $\S 4$ when we endow the space of continuous payoffs with the sup-norm topology considered in $\S 3$ ?

Begin with the first question. Let $\mathscr{U}_{A}^{h}$ denote the space of continuous functions from $A \times \mathscr{M}_{+}^{1}(A, t)$ to $\bar{R}$ endowed with the hypotopology. A natural 
preliminary question to ask is whether the hypotopology is finer than the supnorm topology on the space of continuous payoffs. The answer is no, as can be easily seen by considering a sequence of real-valued functions $\left\{f^{n}\right\}_{n=1}^{\infty}$, $f^{n}:[0,1] \rightarrow[0,1]$ where

$$
\begin{aligned}
f^{n}(x) & =n x, \quad 0 \leq x<1 / n, \\
& =1, \quad 1 / n \leq x \leq 1 .
\end{aligned}
$$

Let $f^{\infty}(x)=1$ for all $0 \leq x \leq 1$. Then $f^{n} \rightarrow f^{\infty}$ in the hypotopology but not in the sup-norm topology. It is also easy to check that the sup-norm topology is finer than the hypotopology; that is, a net $\left\{f^{n}\right\}$ converges to $f$ in the hypotopology if it converges in the sup-norm topology.

We can now ask:

Question. Is Theorem 3.1 valid with $\mathscr{U}_{A}^{h}$ substituted for $\mathscr{U}_{A}$ ?

We do not know the answer to this question. As will emerge from the proofs below, the complete regularity of the space of players and the closed graph property of the correspondence $B_{\tau}$ (see Definition 3.2) play key roles. It is easy to establish the complete regularity of $\mathscr{U}_{A}$ but we could not prove an analog of Claim 4 in the proof of Theorem 3.1 below. To anticipate somewhat, we do not know if $\tau^{\nu} \rightarrow \tau, u^{\nu}(\cdot, \cdot) \stackrel{\text { hypo }}{\rightarrow} u(\cdot, \cdot)$ implies $u^{\nu}\left(\tau^{\nu}, \cdot\right) \stackrel{\text { hypo }}{\rightarrow} u(\tau, \cdot)$ where $\left\{\tau^{\nu}\right\}, \tau$ are chosen from $\mathscr{M}_{+}^{1}(A, t)$ and $\left\{u^{\nu}\right\}, u$ are chosen from $\mathscr{U}_{A}^{h}$.

Since the negative of every lower semicontinuous function is upper semicontinuous, the space of lower semicontinuous functions can be endowed with the "negative" of the hypotopology, the epitopology. Furthermore, since the space of continuous utility functions is both upper and lower semicontinuous, we can endow this space with the smallest topology containing both the epi- and hypotopologies. Dolecki et al. [6] term this the $\pm \mathrm{e}$ topology. It is now natural to ask whether the theory developed in $\S 3$ extends to $\mathscr{U}_{A}^{ \pm \mathrm{e}}$, the space of continuous payoff functions endowed with the $\pm \mathrm{e}$ topology. We can offer

Theorem 5.1. Theorems 3.1, 3.2, and 3.4 are valid with $\mathscr{U}_{A}^{ \pm \mathrm{e}}$ substituted for $\mathscr{U}_{A}$.

We now turn to question (ii). Let $C\left(\mathscr{M}_{+}^{1}(A, t), \mathscr{U}_{A}\right)$ be the space of continuous functions endowed with the compact-open topology. As before, we shall denote this space by $C\left(\mathscr{U}_{A}\right) . C^{u}\left(\mathscr{U}_{A}\right)$ and $C\left(\mathscr{U}_{A}^{h}\right)$ are analogously defined. We can now state

Theorem 5.2. Theorems $3.1,3.2$ and 3.4 are valid with $C\left(\mathscr{U}_{A}\right), C^{u}\left(\mathscr{U}_{A}\right)$, or $C\left(\mathscr{U}_{A}^{h}\right)$ substituted for $\mathscr{U}_{A}$.

We can state, in the context of Theorems 5.1 and 5.2, versions of Theorem 3.3 by focusing on compact subsets of the underlying space of players, but these are not satisfactory because $\mathscr{U}_{A}$ and $\mathscr{U}_{A}^{h}$ are not compact spaces. It would be nice to have esthetically pleasing and economically meaningful conditions 
ensuring compactness of subsets of the various spaces of players considered in Theorem 5.2.

\section{Proofs}

Proof of Theorem 3.1. As in Mas-Colell [16], the proof is an application of the Ky Fan fixed-point theorem (see, for example, Berge [2, p. 251]). We show in a series of claims that all the conditions for the applicability of this theorem are satisfied. Let

$$
\mathscr{T}=\left\{\tau \in \mathscr{M}_{+}^{1}\left(\mathscr{U}_{A} \times A, t\right): \tau_{\mathscr{U}_{A}}=\mu\right\}
$$

Claim 1. $\mathscr{T}$ is nonempty.

Since $A$ is compact, the Riesz representation theorem (see, for example, Berberian [1, Theorem 3, p. 120]) guarantees that we can pick $\nu$ from $\mathscr{M}_{+}^{1}(A, t) \neq \varnothing$. Since $\mu \in \mathscr{M}_{+}^{1}\left(\mathscr{U}_{A}, t\right)$ we can now appeal to Theorem 17 in Schwartz [22, p. 63] to assert the existence of a unique Radon measure $\lambda$ on $A \times \mathscr{U}_{A}$ such that

$$
\lambda(B \times C)=\nu(B) \mu(C) \text { for all } B \in \mathscr{B}(A), C \in \mathscr{B}\left(\mathscr{U}_{A}\right) .
$$

Note that Schwartz states $(*)$ in his theorem in terms of the essential outer measure, but since we are dealing with probability measures, the distinction can be neglected. The reason for this is that we have defined a Radon measure in terms of Definition $R_{3}$ of Schwartz [22, p. 13], and from the proof of $R_{3} \Rightarrow R_{1}$ $[22$, p. 13] we see that the measure of a set with finite measure equals the essential outer measure of that set.

From (*) we obtain

$$
\lambda_{\mathscr{U}_{A}}(B)=\lambda(A \times B)=\nu(A) \mu(B)=\mu(B) \text { for all } B \in \mathscr{B}\left(\mathscr{U}_{A}\right) .
$$

Since $\lambda\left(A \times \mathscr{U}_{A}\right)=\nu(A) \mu\left(\mathscr{U}_{A}\right)=1$ and since, by definition, measures are nonnegative, $\lambda \in \mathscr{T}$ and the proof of the claim is complete.

Claim 2. $\mathscr{T}$ is convex.

Pick $\mu^{1}, \mu^{2}$ from $\mathscr{T}$ and $\lambda$ a real number such that $0<\lambda<1$. Then it is routinely checked that the marginal of $\lambda \mu^{1}+(1-\lambda) \mu^{2}$ on $\mathscr{U}_{A}$ is $\mu$ and that $\lambda \mu^{1}+(1-\lambda) \mu^{2} \in \mathscr{M}_{+}^{1}\left(\mathscr{U}_{A} \times A\right)$.

Claim 3. $\mathscr{T}$ is compact.

Pick a net $\left\{\gamma^{\alpha}\right\}$ from $\mathscr{T}$. We first show that the marginal of $\gamma^{\alpha}$ on $A, \gamma_{A}^{\alpha}$, is an element of $\mathscr{M}_{+}^{1}(A, t)$. Let $p_{A}$ be the projection map from $A \times \mathscr{U}_{A}$ to $A$. Then $\gamma_{A}^{\alpha}$ can be written as the image measure $p_{A} \cdot \gamma^{\alpha}=\gamma^{\alpha} \cdot p_{A}^{-1}$. Since $p_{A}$ is a continuous function and we are working with probability measures, the image measure is Radon [22, last paragraph on p. 36].

Since $\left\{\gamma_{A}^{\alpha}\right\}$ is a net chosen from $\mathscr{M}_{+}^{1}(A, t)$, we can appeal to Theorem 2.2 to assert the existence of a subnet, indicated by $\left\{\gamma_{A}^{\beta}\right\}$, such that $\gamma_{A}^{\beta} \rightarrow \gamma_{A}$ and $\gamma_{A} \in \mathscr{M}_{+}^{1}(A, t)$. Since $A$ is compact, $A$ is completely regular (see, for example, Willard [25, p. 95, Corollary 15.7, Theorem 17.10]). Again, since $\mathscr{U}_{A}$ is metric, 
it is completely regular (see, for example, [25, Example 14.9]). We can now appeal to Theorem 2.6, to assert the existence of a measure $\gamma \in \mathscr{M}_{+}^{1}\left(A \times \mathscr{U}_{A}\right)$ such that

(i) $\gamma_{\mathscr{U}_{A}}=\mu$;

(ii) $\gamma^{\beta} \rightarrow \gamma$.

This complete the proof of the claim.

Claim 4. $B: \mathscr{T} \rightarrow 2^{A \times \mathscr{U}_{A}}$ has a closed graph.

Let $\tau^{\nu} \rightarrow \tau,\left(u^{\nu}, a^{\nu}\right) \rightarrow(u, a)$, and $\left(u^{\nu}, a^{\nu}\right) \in B_{\tau^{\nu}}$. Suppose $(u, a) \notin B_{\tau}$. Then there exists $\hat{x} \in A$ such that

$$
u\left(\hat{x}, \tau_{A}\right)>u\left(a, \tau_{A}\right) .
$$

Hence there exists $\varepsilon>0$ such that

$$
u\left(\hat{x}, \tau_{A}\right)>u\left(a, \tau_{A}\right)+\varepsilon .
$$

Since $\tau^{\nu} \rightarrow \tau$, Theorem 2.5 yields that $\tau_{A}^{\nu} \rightarrow \tau_{A}$, and since $u$ is jointly continuous on $A \times \mathscr{M}_{+}^{1}(A, t)$, there exists $\bar{\nu}$ such that

$$
\left|u\left(\hat{x}, \tau_{A}\right)-u\left(\hat{x}, \tau_{A}^{\nu}\right)\right|<\varepsilon / 4 \text { for all } \nu>\bar{\nu} \text {. }
$$

By the same reasoning, there exists $\overline{\bar{\nu}}$ such that

$$
\left|u\left(a, \tau_{A}\right)-u\left(a^{\nu}, \tau_{A}^{\nu}\right)\right|<\varepsilon / 4 \text { for all } \nu>\bar{\nu} .
$$

This implies that

$$
\begin{aligned}
& u\left(\hat{x}, \tau_{A}^{\nu}\right)>u\left(\hat{x}, \tau_{A}\right)-\varepsilon / 4 \\
& u\left(a, \tau_{A}\right)>u\left(a^{\nu}, \tau_{A}^{\nu}\right)-\varepsilon / 4
\end{aligned}
$$

On substituting in $(\dagger)$ we obtain

$$
u\left(\hat{x}, \tau_{A}^{\nu}\right)+\varepsilon / 4>u\left(a^{\nu}, \tau_{A}^{\nu}\right)+\varepsilon-\varepsilon / 4 \text { for all } \nu>\operatorname{Max}(\bar{\nu}, \overline{\bar{\nu}}) \equiv \nu^{\prime} .
$$

This can be simplified to yield

$$
u\left(\hat{x}, \tau_{A}^{\nu}\right)>u\left(a^{\nu}, \tau_{A}^{\nu}\right)+\varepsilon / 2 \text { for all } \nu>\nu^{\prime} .
$$

Since $\mathscr{U}_{A}$ is endowed with the sup norm topology, there exists $\nu^{\prime \prime}$ such that for all $z \in A \times \mathscr{M}_{+}^{1}(A, t)$,

$$
\left|u^{\nu}(z)-u(z)\right|<\varepsilon / 4 \text { for all } \nu>\nu^{\prime \prime}
$$

Let $\nu_{0}>\operatorname{Max}\left(\nu^{\prime}, \nu^{\prime \prime}\right)$ and deduce

$$
\begin{aligned}
& u^{\nu_{0}}\left(\hat{x}, \tau_{A}^{\nu_{0}}\right)>u\left(\hat{x}, \tau_{A}^{\nu_{0}}\right)-\varepsilon / 4 \\
& u\left(a^{\nu_{0}}, \tau_{A}^{\nu_{0}}\right)>u^{\nu_{0}}\left(a^{\nu_{0}}, \tau_{A}^{\nu_{0}}\right)-\varepsilon / 4
\end{aligned}
$$

On substituting in $(\dagger \dagger)$, we obtain

$$
u^{\nu_{0}}\left(\hat{x}, \tau^{\nu_{0}}\right)+\varepsilon / 4>u^{\nu_{0}}\left(a^{\nu_{0}}, \tau^{\nu_{0}}\right)-\varepsilon / 4+\varepsilon / 2
$$

This simplifies to yield a contradiction to the fact that $\left(u^{\nu_{0}}, a^{\nu_{0}}\right) \in B_{\tau^{\nu_{0}}}$. This contradiction proves the claim. 
Claim 5. For any $\tau \in \mathscr{T}, B_{\tau}$ is a closed set in $\mathscr{U}_{A} \times A$.

This is a simple consequence of Claim 4.

Next, we consider the map $Q: \mathscr{T} \rightarrow 2^{\mathscr{T}}$ such that

$$
Q(\tau)=\left\{\rho \in \mathscr{T}: \rho\left(B_{\tau}\right)=1\right\} .
$$

Claim 6. For any $\tau \in \mathscr{T}, Q(\tau) \neq \varnothing$.

From Theorem 2.7, we know that there exists a net $\left\{\mu^{\nu}\right\}$ converging to $\mu$ and such that each $\mu^{\nu}$ has a finite support. Pick a particular $\mu^{\nu}$ and assume that its support consists of $k$ elements, $u_{1}, \ldots, u_{k}$. Let

$$
S\left(u_{i}\right)=\left\{a \in A: u_{i}\left(a, \tau_{A}\right) \geq u_{i}\left(A, \tau_{A}\right)\right\} .
$$

Certainly, $S\left(u_{i}\right)$ is a closed, and hence compact, subset of $A$. As in Claim 1, the Riesz representation theorem guarantees an element $\bar{\nu}_{i}$ in $\mathscr{M}_{+}^{1}\left(S\left(u_{i}\right), t\right)$. We can extend $\bar{\nu}_{i}$ to $\mathscr{B}(A)$ by defining for each $W \in \mathscr{B}(A)$,

$$
\nu_{i}(W)=\bar{\nu}_{i}\left(S\left(u_{i}\right) \cap W\right) .
$$

Since $S\left(u_{i}\right)$ is a closed subset of $A$, certainly $\left(S\left(u_{i}\right) \cap W\right) \in \mathscr{B}\left(S\left(u_{i}\right)\right)$ and hence $\nu_{i}$ is well defined (see, for example, Berberian [1, Exercise 17 on $\mathrm{p}$. 183]). It can also be easily checked that $\nu_{i}$ is a measure. Certainly

$$
\nu_{i}(A)=\bar{\nu}_{i}\left(S\left(u_{i}\right) \cap A\right)=\bar{\nu}_{i}\left(S\left(u_{i}\right)\right)=1 .
$$

Furthermore, for any disjoint countable family $\left\{W_{i}\right\}$ chosen from $\mathscr{B}(A)$,

$$
\begin{aligned}
\nu_{i}\left(\bigcup_{i} w_{i}\right) & =\bar{\nu}_{i}\left(S\left(u_{i}\right) \cap \bigcup_{i} W_{i}\right)=\bar{\nu}_{i}\left(\bigcup_{i}\left(S\left(u_{i}\right) \cap W_{i}\right)\right) \\
& =\sum_{i} \bar{\nu}_{i}\left(S\left(u_{i}\right) \cap W_{i}\right)=\sum_{i} \nu_{i}\left(W_{i}\right) .
\end{aligned}
$$

Finally, to show that $\bar{\nu}_{i}$ is Radon, pick any $W_{i} \in \mathscr{B}(A)$ and such that $\nu_{i}\left(W_{i}\right)>$ 0 . This means that $\bar{\nu}_{i}\left(S\left(u_{i}\right) \cap W_{i}\right)>0$. But $\bar{\nu}_{i}$ is Radon and hence for any positive $\varepsilon$ there exists a compact set $K \subset\left(S\left(u_{i}\right) \cap W_{i}\right)$ such that $\bar{\nu}_{i}(K)>$ $\bar{\nu}_{i}\left(S\left(u_{i}\right) \cap W_{i}\right)-\varepsilon$. This implies that $\nu_{i}(K)>\nu_{i}\left(W_{i}\right)-\varepsilon$ and we are done.

Now for any $W \in \mathscr{B}(A) \times \mathscr{B}\left(\mathscr{U}_{A}\right)$ define

$$
\rho^{\nu}(W)=\sum_{i=1}^{k} \nu_{i}\left(W_{u_{i}}\right) \mu^{\nu}\left(u_{i}\right),
$$

where $W_{u_{i}}=\left\{a \in A:\left(a, u_{i}\right) \in W\right\}$. Certainly the sections $W_{u_{i}}$ are measurable (see, for example, [1, Theorem 3, p. 120]) and hence $\rho^{\nu}$ is well defined. To show that $\rho^{\nu}$ is a measure, pick a countable family of disjoint subsets $W_{j}$ from $\mathscr{B}(A) \times \mathscr{B}\left(\mathscr{U}_{A}\right)$. The second equality in the following demonstration follows from Theorem 2 on p. 120 in [1]; the third equality relies on $\nu_{i}$ being measures 
and on $\left(W_{j}\right)_{u_{i}}$ and $\left(W_{k}\right)_{u_{i}}, j \neq k$, being disjoint.

$$
\begin{aligned}
\rho^{\nu}\left(\bigcup_{j} W_{j}\right) & =\sum_{i=1}^{k} \nu_{i}\left(\left(\bigcup_{j} W_{j}\right)_{u_{i}}\right) \mu^{\nu}\left(u_{i}\right)=\sum_{i=1}^{k} \nu_{i}\left(\bigcup_{j}\left(W_{j}\right)_{u_{i}}\right) \mu^{\nu}\left(u_{i}\right) \\
& =\sum_{i=1}^{k}\left(\sum_{j=1}^{\infty}\left(W_{j}\right)_{u_{i}}\right) \mu^{\nu}\left(u_{i}\right)=\sum_{j=1}^{\infty} \rho^{\nu}\left(W_{j}\right) .
\end{aligned}
$$

Next, to show that $\rho^{\nu}$ is Radon, pick $W \in \mathscr{B}(A) \times \mathscr{B}\left(\mathscr{U}_{A}\right)$ such that $\rho^{\nu}>0$. Now for any $\varepsilon>0$ we have to find a compact set $K \subset W$ such that $\rho^{\nu}(K)>$ $\rho^{\nu}(W)-\varepsilon$. Since $\sum_{i=1}^{k} \nu_{i}\left(W_{u_{i}}\right) \mu^{\nu}\left(u_{i}\right)>0$, without any loss of generality we can assume $\nu_{i}\left(W_{u_{i}}\right)>0$ for all $i$. Since $\nu_{i}$ are Radon, we can find compact $K_{i} \subset W_{u_{i}}$ such that

$$
\nu_{i}\left(K_{i}\right)>\nu_{i}\left(W_{u_{i}}\right)-\varepsilon .
$$

Let $K=\bigcup_{i=1}^{k} K_{i}$. Certainly $K$ is compact. Since $K_{i} \cap K_{j}=\varnothing$ for $i \neq j$,

$$
\rho^{\nu}(K)=\sum_{i=1}^{k} \nu_{i}\left(K_{i}\right) \mu^{\nu}\left(u_{i}\right)>\rho^{\nu}(W)-\varepsilon .
$$

Finally, we check that $\rho_{\mathscr{U}_{A}}^{\nu}=\mu^{\nu}$. This is easy because for any $W \in$ $\mathscr{B}\left(\mathscr{U}_{A}\right), \rho_{A}^{\nu}(W)=\rho^{\nu}(A \times W)=\sum_{i \in I} \nu_{i}(A) \mu^{\nu}\left(u_{i}\right)=\mu^{\nu}(W)$ where $I=\{i \in$ $\left.(1,2, \ldots, k): u_{i} \in W\right\}$.

Since $\mathscr{M}_{+}^{1}(A, t)$ is compact by virtue of Theorem 2.3 , there exists a convergent subnet $\rho_{A}^{\alpha}$ with limit $\gamma_{A}$. By construction we know that $\rho_{\mathscr{U}_{A}}^{\alpha}=\mu^{\alpha}$, and by hypothesis $\mu^{\alpha}$ has limit $\mu$. We now appeal to Theorem 2.6 to assert the existence of a limit point $\rho$ of $\rho^{\alpha}$ in $\mathscr{M}_{+}^{1}\left(A \times \mathscr{U}_{A}, t\right)$ such that $\rho_{A}=\gamma_{A}$ and $\rho_{\mathscr{U}_{A}}=\mu$. All that remains to be shown is that $\rho\left(B_{\tau}\right)=1$. But by Theorem 2.4,

$$
\rho\left(B_{\tau}\right) \geq \lim \sup \rho^{\alpha}\left(B_{\tau}\right)=1 \text {. }
$$

Since $\rho\left(A \times \mathscr{U}_{A}\right)=\lim \rho^{\alpha}\left(A \times \mathscr{U}_{A}\right)=1$, we are done.

Remark. In the case when $A$ is compact metric, and hence separable, one has an alternative proof of Claim 6 that does not hinge on Theorem 2.7. Consider $\Phi(u)=\left\{a \in A:(a, u) \in B_{\tau}\right\}$, the $u$-section of $B_{\tau}$ on $A$. Certainly $\Phi(u) \neq$ $\varnothing$ for all $u \in \mathscr{U}_{A}$ and, given Claim $5, \Phi$ has a measurable graph. Hence by Aumann's selection theorem (see Castaing-Valadier [4, Theorem 3.22]) a measurable selection $h: \mathscr{U}_{A} \rightarrow A$. Since $A$ is separable metric, $h$ is Lusin measurable; see Schwartz [22, Theorem 5, p. 26]. Let $f: \mathscr{U}_{A} \rightarrow A \times \mathscr{U}_{A}$ be defined by $f(u)=(h(u), u)$. Since $\mu$ is a finite probability measure, certainly $f$ is $\mu$-proper [22, Definition 10, p. 31] and hence the image meaure $f \mu$ is Radon [22, p. 32]. It is now easy to check that $f \mu\left(B_{\tau}\right)=1$ and that $(f \mu)_{\mathscr{U}_{A}}$, the marginal of $f \mu$ on $\mathscr{U}_{A}$, is $\mu$. Hence $f \mu \in \mathscr{T}$ and we are done. This proof does not work in our generalized setup because of the difficulty of finding Lusin measurable selections. 
Claim 7. For any $\tau \in \mathscr{T}, Q(\tau)$ is convex.

This is straightforward.

Before considering our next claim, we develop some lemmata. For the definition and properties of the limsup of a sequence of sets, see, for example, Klein-Thompson [12].

Lemma 1. Let $\left\{A_{\nu}\right\}$ be a net of subsets of a compact set $K$ and such that $\lim \sup A_{\nu} \subset W$ for an open set $W$. Then for all $\bar{\nu}$ there exists $\nu>\bar{\nu}$ such that $A_{\nu} \subset W$.

Proof. Suppose not. Then there exists $\bar{\nu}$ such that for al $\nu>\bar{\nu} A_{\nu} \not \subset W$, that is, $A_{\nu} \cap W^{c} \neq \varnothing$. Pick $x^{\nu} \in A_{\nu} \cap W^{c}$ and manufacture a subset $\left\{x^{\nu}\right\}$. Since this subnet lies in $K$, there exist a further subnet $\left\{x^{\nu \alpha}\right\}$ and $\bar{x} \in K$ such that $x^{\nu \alpha} \rightarrow \bar{x}$. Since $x^{\nu \alpha} \in W^{c}$ for all $\alpha$ and since $W^{c}$ is closed, $\bar{x} \in W^{c}$. Furthermore, $\bar{x} \in \lim \sup A_{\nu}$. Hence

$$
\lim \sup A_{\nu} \cap W^{c} \neq \varnothing,
$$

a contradiction which proves the lemma.

Lemma 2. For any normal topological space $X$, let $\{\tau\}$ be a net chosen from $\mathscr{M}_{+}^{1}(X, t), \tau_{\nu} \rightarrow \tau$, and $\left\{A_{\nu}\right\}$ a net of subsets of a compact set $K$ such that $\tau_{\nu}\left(A_{\nu}\right) \geq m$ for all $\nu$ and for some $m>0$. Then $\tau\left(\lim \sup A_{\nu}\right) \geq m$.

Proof. Denote $\limsup A_{\nu}$ by $\bar{A}$. Suppose $\tau(\bar{A})<m$; that is, there exists $\delta>0$ such that $\tau(\bar{A})<m-\delta$. This implies that $\tau\left(A^{c}\right)>1-m+\delta$. Since $\tau \in \mathscr{M}_{+}^{1}(X, t)$, there exists a compact set $L$ such that $L \subset \bar{A}^{c}$

$$
\tau(L)>1-m+(\delta / 2) .
$$

Since $X$ is normal, there exist open sets $V$ and $W$ such that $V \cap W=\varnothing$ and $\bar{A} \subset W$ and $L \subset V$.

By monotonicity of measures, $\tau(V) \geq \tau(L)>1-m+(\delta / 2)$. Hence $\tau\left(V^{c}\right) \leq$ $m-(\delta / 2)$. Since $V^{c}$ is a closed set, we can appeal to Theorem 2.4 to assert that $\lim \sup \tau_{\nu}\left(V^{c}\right) \leq \tau\left(V^{c}\right)$. Hence there exist a subnet $\left\{\tau_{\rho}\right\}$ and $\bar{\rho}$ such that for all $\rho>\bar{\rho}$,

$$
\tau_{\rho}\left(V^{c}\right) \leq \tau\left(V^{c}\right)+(\delta / 4) .
$$

Since $\limsup A_{\rho} \subset \bar{A} \subset W$, we can appeal to Lemma 1 to find a $\rho^{\prime}>\bar{\rho}$ such that $A_{\rho^{\prime}} \subset W$. Hence $A_{\rho^{\prime}} \subset V^{c}$. Again by monotonicity of measures, $\tau_{\rho^{\prime}}\left(A_{\rho^{\prime}}\right) \leq m-(\delta / 4)$, a contradiction which proves the lemma.

Lemma 3. $\mathscr{U}_{A} \times A$ is a normal space.

Proof. $A$ is compact Hausdorff, and hence $\sigma$-compact and regular Hausdorff. Since $\mathscr{U}_{A}$ is a metric space, we can appeal to a result of Michael (see Morita $[17$, p. 25 , Assertion 3]) to complete the proof of the lemma.

Claim 8. $Q$ is an upper hemicontinouous correspondence. 
Since $\mathscr{T}$ is compact, it suffices to show that $Q$ has a closed graph (see [2, p. 112, Corollary]). Toward this end, let $\tau^{\nu} \rightarrow \tau, \rho^{\nu} \in Q\left(\tau^{\nu}\right), \rho^{\nu} \rightarrow \rho$. Assume that $\rho \notin Q(\tau)$, that is, $\rho\left(B_{\tau}\right)<1$. Hence there exists $\varepsilon>0$ such that $\rho\left(B_{\tau}\right)<1-\varepsilon$.

Since $\nu$ is a Radon probability on $\mathscr{U}_{A}$, there exists a compact subset of $\mathscr{U}_{A}$ such that $\mu(M)>1-(\varepsilon / 4)$. Since $\left\{\rho^{\nu}\right\}$ and $\rho$ are in (recall that $\mathscr{T}$ is compact and hence closed), certainly

$$
\begin{aligned}
\rho^{\nu}(A \times M) & =\mu(M)=1-(\varepsilon / 4) \text { for all } \nu, \\
\rho(A \times M) & =\mu(M)=1-(\varepsilon / 4) .
\end{aligned}
$$

Since $A$ is compact, $M \times A$ is a compact set in the product topology. Call it $K$. Now let

$$
B_{\tau^{\nu}}^{K}=B_{\tau^{\nu}} \cap K \text { for all } \nu .
$$

We claim that $\rho^{\nu}\left(B_{\tau^{\nu}}^{K}\right)>1-(\varepsilon / 2)$ for each $\nu$. Suppose not. Then for some $\nu, \rho^{\nu}\left(B_{\tau^{\nu}}^{K}\right) \leq 1-(\varepsilon / 2)$. But $\rho^{\nu}\left(B_{\tau^{\nu}}\right)=1$ by hypothesis. Hence there exists a measurable subset $Q$ of $B_{\tau^{\nu}}$ such that $Q \cap K=\varnothing$ and $\rho^{\nu}(Q) \geq(\varepsilon / 2)$. But this contradicts the fact that $\rho^{\nu}(K)=1-(\varepsilon / 4)$.

By virtue of Lemma 3, we can appeal to Lemma 2 to conclude that

$$
\rho\left(\lim \sup B_{\tau^{\nu}}^{K}\right) \geq 1-(\varepsilon / 2) .
$$

We can now appeal to Claim 4 to assert that

$$
\lim \sup \left(B_{\tau^{\nu}}^{K}\right) \subset B_{\tau}^{K} .
$$

Since $B_{\tau}^{K} \subset B_{\tau}$, monotonicity of a measure yields

$$
\rho\left(B_{\tau}\right) \geq 1-(\varepsilon / 2),
$$

a contradiction which completes the proof of the claim.

We can now apply the Ky Fan fixed-point theorem (see, for example, [2, p. 251]) to the map $Q$ to complete the proof of the theorem.

Proof of Corollary. This is a direct consequence of Theorem 2.9 and Theorem 3.1.

Proof of Theorem 3.2. As in Claim 8, it suffices to show that $\Gamma$ has a closed graph. Toward that end, let $\mu^{\nu} \rightarrow \mu, \tau^{\nu} \in \Gamma\left(\mu^{\nu}\right), \tau^{\nu} \rightarrow \tau$. We have to show that

(i) $\tau_{\mathscr{U}_{A}}=\mu$,

(ii) $\tau\left(B_{\tau}\right)=1$.

(i) is a consequence of Theorem 2.5. To show (ii) we repeat the arguments of the proof of Claim 8 with $\left\{\tau^{\nu}\right\}$ substituted for the net $\left\{\rho^{\nu}\right\}$.

Proof of Theorem 3.3. By the Ascoli-Arzela theorem (see, for example, [18, p. 283]), $\mathcal{J}$ is compact. Hence the range space of $\Gamma$ is compact by virtue of Theorem 2.3. We now appeal to the fact that a mapping with a closed graph 
is upper hemicontinuous if the range space is compact; see, for example, [2, p. 251].

Proof of Theorem 3.4. The first claim is a direct consequence of Theorem 2.8 once we recall that the Borel and Baire $\sigma$-algebras are identical on a metric space and that every Baire measure on a compact Hausdorff space is tight. The second claim then follows from Theorem 3.1.

Proof of Theorem 4.1. This follows directly from Dugundji [7, Theorem 3.1, p. 261] once we observe from Theorem 2.3 that $\mathscr{M}_{+}^{1}(A, t)$ is compact and hence locally compact.

Proof of Theorem 4.2. Since $A$ is Hausdorff compact by hypothesis and $\mathscr{M}_{+}^{1}(A, t)$ is Hausdorff compact by virtue of Theorems 2.2 and $2.3, A \times \mathscr{M}_{+}^{1}(A, t)$ is a Hausdorff compact space. We can now appeal to Corollary 4.3 in Dolecki et al. [6] to complete the proof.

Proof of Theorem 4.3. Consider first the proof of Theorem 3.1 with $C\left(\mathscr{S}_{A}^{h}\right)$ substituted for $\mathscr{U}_{A}$. We first show the following.

Claim 1. $C\left(\mathscr{S}_{A}^{h}\right)$ is a completely regular space.

Since $\mathscr{S}_{A}^{h}$ is Hausdorff compact by virtue of Theorem 4.2, it is normal and hence completely regular. We can now appeal to Nagata [18, p. 272] or Willard $[25,43 \mathrm{~B} 3$, p. 288$]$ to complete the proof of the claim.

We used the property of complete regularity of $\mathscr{U}_{A}$ in the proofs of Claims 3 and 6 . In the proof of Claim 8, we used normality of $\mathscr{U}_{A} \times A$ in the proof of Lemma 2 and established this normality property in Lemma 3. However, inspection of the proof of Lemma 2 reveals that of the two sets $\bar{A}$ and $L$ to be separated by disjoint open sets, one of the sets, $L$, is compact. Hence the normality property is much stronger than what is needed; in particular, regularity of $\mathscr{U}_{A} \times A$ would have sufficed (see Dugundji [7, XI-1.5(c)] or Willard [25, Lemma 43.3]). Since a product of completely regular spaces is completely regular [see, for example, [7, VII-7.2(2)]), we have established the following:

Claim 2. Lemma 2 is valid for the space $A \times C\left(\mathscr{S}_{A}^{h}\right)$ substituted for the space $X$.

All that remains is a proof of the analog of Claim 4. Before we turn to this, we develop two lemmata that may have independent interest.

Lemma 4. Let $\left\{f^{\nu}\right\}$ be a net of upper semicontinuous functions from a compact Hausdorff space $X$ into $\bar{R}$ and such that $f^{\nu}$ hypoconverges to $f$. Then the following is true for any $x$ in $X$ :

(i) for all $x^{\nu} \rightarrow x, \lim \sup f^{\nu}\left(x^{\nu}\right) \leq f(x)$,

(ii) there exists $x^{\nu} \rightarrow x, \liminf f^{\nu}\left(x^{\nu}\right) \geq f(x)$.

Proof. We begin with (i). Pick any $\delta>0$ and consider $(x, f(x)+\delta)$. Certainly, it is not in hypograph $f$. Since hypograph $f$ is a closed subset of $X \times R$, there exists an open neighborhood of $(x, f(x)+\delta)$ which is disjoint from hypograph 
$f$. Since $X \times R$ is regular (see, for example, [7, IX-5.2, VII-2.3(2), VII-2.2(2)]), there exists $\varepsilon>0$ and an open neighborhood $V$ of $x$ such that

$$
\bar{V} \times \overline{B_{\varepsilon}(f(x)+\delta)} \cap \text { hypograph } f=\varnothing,
$$

where $\bar{V}$ denotes the closure of $V$ and $B_{\varepsilon}(x)$ is an open ball with radius $\varepsilon$ and center $x$. Since $\bar{V}$ is compact, by the definition of the topology of closed convergence, there exists $\bar{\nu}$ such that

$$
\bar{V} \times \overline{B_{\varepsilon}(f(x)+\delta)} \cap \text { hypograph } f^{\nu}=\varnothing \text { for all } \nu>\bar{\nu} .
$$

Since $x^{\nu} \rightarrow x$, there exists $\overline{\bar{\nu}}$ such that $x^{\nu} \in V$ for $\nu>\bar{\nu}$. Let $\nu^{\prime}>(\bar{\nu}, \bar{\nu})$. Certainly, for all $\nu>\nu^{\prime}, f^{\nu}\left(x^{\nu}\right) \notin B_{\varepsilon}(f(x)+\delta)$. We now claim that $f^{\nu}\left(x^{\nu}\right)<$ $f(x)+\delta-\varepsilon$; if not, we obtain a contradiction to $(*)$. Thus, there exists $\nu^{\prime}$ such that for all $\nu>\nu^{\prime}, f^{\nu}\left(x^{\nu}\right)<f(x)+\delta$. But $\delta$ was arbitrary and therefore

$$
\lim \sup f^{\nu}\left(x^{\nu}\right) \leq f(x)
$$

and (i) is proved.

We now turn to the proof of (ii). Since $(x, f(x)) \in$ hypograph $f$, by the definition of the topology of closed convergence, for any $\varepsilon>0$ and any open neighborhood $W$ of $x$, there exists $\bar{\nu}$ such that $\nu>\bar{\nu}$ implies

$$
\left(W_{x} \times B_{\varepsilon}(f(x))\right) \cap \text { hypograph } f^{\nu} \neq \varnothing .
$$

Pick $\left(x^{\nu}, z^{\nu}\right) \in$ hypograph $f^{\nu}$ such that $x^{\nu} \in W_{u}$ and $z^{\nu} \in B_{\varepsilon}(f(x))$. We can thus manufacture a net $\left\{x^{\nu}, z^{\nu}\right\}$ such that $x^{\nu} \rightarrow x, z^{\nu} \leq f^{\nu}\left(x^{\nu}\right)$, and $z^{\nu} \rightarrow f(x)$. But this implies that

$$
\liminf f^{\nu}\left(x^{\nu}\right) \geq \liminf z^{\nu}=f(x)
$$

and (ii) is proved.

Remark. Lemma 2 is a generalization, in one direction, of a well-known characterization of a hypoconvergent sequence of upper semicontinuous functions on a locally compact Hausdorff space that admits a countable base; see, for example, Salinetti-Wets [20, Theorem 2.4].

Lemma 5. Let $\left\{f^{\nu}\right\}$ and $f$ be as in Lemma 4. If $x^{\nu} \in \operatorname{Arg} \operatorname{Max}_{x \in X} f^{\nu}(x)$ and $x^{\nu} \rightarrow x$, then $x \in \operatorname{Arg} \operatorname{Max}_{x \in X} f(x)$.

Proof. Suppose the conclusion is false. Then there exists $\hat{x} \in X$ such that $f(\hat{x})>f(x)$. Now appeal to Lemma 4 to construct a net $\left\{\hat{x}^{\nu}\right\}$ in $X, \hat{x}^{\nu} \rightarrow \hat{x}$,

$$
\liminf f^{\nu}\left(\hat{x}^{\nu}\right) \geq f(\hat{x})>f(x) \geq \limsup f^{\nu}\left(x^{\nu}\right) .
$$

But $f^{\nu}\left(x^{\nu}\right) \geq f^{\nu}\left(\hat{x}^{\nu}\right)$. Hence

$$
\lim \sup f^{\nu}\left(x^{\nu}\right) \geq \limsup f^{\nu}\left(\hat{x}^{\nu}\right) \geq \liminf f^{\nu}\left(\hat{x}^{\nu}\right),
$$

a contradiction.

Remark. Lemma 5 is also a standard result in the theory of variational convergence, as, for example, in Wets [24, Theorem 3]. However, we could not find any reference to the result in a set-up such as ours. 
We can now state

Claim 3. $B: \mathscr{T} \rightarrow 2^{A \times C\left(\mathscr{S}_{A}^{h}\right)}$ has a closed graph.

Let $\tau^{\nu} \rightarrow \tau, f^{\nu} \rightarrow f$, and $a^{\nu} \rightarrow a$, where $\left\{\tau^{\nu}\right\}$ is a net in $\mathscr{T},\left\{f^{\nu}\right\}$ is a net in $C\left(\mathscr{S}_{A}^{h}\right),\left\{a^{\nu}\right\}$ a net in $A$, and $\left(a^{\nu}, f^{\nu}\right) \in B_{\tau \nu}$. We have to show that $(a, f) \in B_{\tau}$.

By Theorem 2.5, we know that $\tau_{A}^{\nu} \rightarrow \tau_{A}$. Furthermore, since we are working with the compact-open topology, the evaluation map is continuous; see Dugundji [7, XII-2.4(1)]. Hence $f^{\nu}\left(\tau_{A}^{\nu}\right) \rightarrow f\left(\tau_{A}\right)$. Since

$$
a^{\nu} \in \operatorname{Arg} \operatorname{Max}_{x \in A}\left[f^{\nu}\left(\tau_{A}^{\nu}\right)\right](x), \quad a^{\nu} \rightarrow a .
$$

Lemma 5 implies $a \in \operatorname{Arg} \operatorname{Max}_{x \in A}\left[f\left(\tau_{A}^{\prime}\right)\right](x)$, and we are done.

We now have all the steps that we need to construct a proof of Theorem 3.1 with $C\left(\mathscr{S}_{A}^{h}\right)$ substituted for $\mathscr{U}_{A}$. The proof of the corresponding analogs of Theorems 3.2 and 3.4 are routine. All that remains is a proof of an analog of Theorem 3.3, but for this we simply use Theorem 4.2 on the compactness of $\mathscr{S}_{A}^{h}$ coupled with the generalization of the Ascoli-Arzela theorem given in Nagata [18, Theorem VII-1, p. 280].

The proof of Theorem 4.3 is now complete.

Proof of Theorem 4.4. The proof is essentially the observation that the compactopen topology coincides with the topology of uniform convergence when $\mathscr{S}_{A}^{h}$ is compact; see, for example, [18, p. 277]. The fact that $\mathscr{S}_{A}^{h}$ is compact follows from Theorem 4.2.

Proof of Theorem 4.5. The proof of Theorem 4.3 revolves around two basic points. The first is the complete regularity of $C\left(\mathscr{S}_{A}^{h}\right)$ as established in Claim 1 and used in Claim 2. The second aspect consists of the basic property of hypoconvergence as established in Lemmata 4 and 5 and used in Claim 3. Since $\mathscr{S}_{A}^{h}$ and $\mathscr{S}_{A}^{h}(b)$ are subspaces of $\mathscr{S}_{A}^{h}$, they are completely regular; see, for example, [7, VII, Theorem 7.2 (1), p. 154]. We can appeal to Nagata or Willard to assert the complete regularity of $C\left(\mathscr{S}_{A}^{h}\right)$ and $C\left(\mathscr{S}_{A}^{h}(b)\right)$. We leave it to the reader to check that nothing need be changed in the proofs of Lemmata 4 and 5.

The compactness of $\mathscr{S}_{A}^{h}(b)$ follows from Dolecki et al. [6, Corollary 4.4]. The validity of Theorem 4.4 for $C^{u}\left(\mathscr{S}_{A}^{h}(b)\right)$ is now routine.

Proof of Theorem 5.1. The reader can check that is only the proof of Theorem 3.1 that does not routinely extend to the space $\mathscr{U}_{A}^{ \pm \mathrm{e}}$ and we confine ourselves to it. We first establish the following:

Claim 1. $\mathscr{U}_{A}^{ \pm \mathrm{e}}$ is a completely regular space.

The space of real-valued continuous functions on $A \times \mathscr{M}_{+}^{1}(A, t)$ endowed with the hypotopology is a subspace of a compact Hausdorff space by virtue of Theorem 4.2. As such, it is completely regular. Similarly, the space of continuous functions on $A \times \mathscr{M}_{+}^{1}(A, t)$ endowed with the epitopology is a completely 
regular space. We now appeal to Willard [25, 13H, p. 90] to assert the validity of the claim.

Just as in the proof of Theorem 4.3, we can now establish the following:

Claim 2. Lemma 2 is valid for the space $A \times \mathscr{U}_{A}^{ \pm \mathrm{e}}$ substituted for the space $X$.

All that remains is a proof of the analog of Claim 4 in the proof of Theorem 3.1. But this follows from

Claim 3. The $\pm \mathrm{e}$ topology on the space of real-valued continuous functions on $A \times \mathscr{M}_{+}^{1}(A, t)$ is finer than the sup-norm topology on the same space.

Given Theorem 2.3, the claim is a direct consequence of an observation of Dolecki et al. [6, p. 427, paragraph 6].

The proof of Theorem 5.2 involves no new ideas and we leave it to the reader, except for one fact which is needed to establish the proof of an analog of Claim 4 in the proof of Theorem 3.1.

Lemma 6. Let $X$ be a compact Hausdorff space and $C(X)$ the space of continuous real-valued functions on $X$. Then for any net $\left\{f^{\nu}\right\}$ and $f$ chosen from $C(X)$, the convergence of $f^{\nu}$ to $f$ in the sup-norm topology implies this convergence in the hypotopology.

Proof. We have to show the convergence of hypograph $f^{\nu}$ to hypograph $f$ in the topology of closed convergence on $X \times R$. Given Definition 3.1.4 and Theorem 4.5.4 in Klein-Thompson [12], we have to show that the set of limit points and cluster points of a net $\left(x^{\nu}, z^{\nu}\right)$ chosen from hypograph $f^{\nu}$ belongs to hypograph $f$.

To establish this, let $\left(x^{\nu}, z^{\nu}\right)$ converge to $(x, z)$. Since $\left(x^{\nu}, z^{\nu}\right)$ belongs to hypograph $f^{\nu}$, certainly $z^{\nu} \leq f^{\nu}\left(x^{\nu}\right)$. Since

$$
\left|f^{\nu}\left(x^{\nu}\right)-f(x)\right| \leq\left|f^{\nu}\left(x^{\nu}\right)-f\left(x^{\nu}\right)\right|+\left|f\left(x^{\nu}\right)-f(x)\right|
$$

it is easy to establish that $f^{\nu}\left(x^{\nu}\right)$ tends to $f(x)$. Hence $z \leq f(x)$ and therefore $(z, x) \in$ hypograph $f$.

The proof for cluster points is identical; we simply work with the relevant subnet.

\section{ACKNOWLEDGEMENT}

A first version of this paper was circulated as BEBR Faculty Paper No. 1306 under the title On a variant of a theorem of Schmeidler. I am grateful to Ye Neng Sun for finding a gap in the proof of Theorem 3.1 in this version. I also thank Tapan Mitra for several discussions over the last three years and an anonymous referee for her/his careful reading. Errors are, of course, solely mine. This research was presented at the Mathematical Economics Workshop at Indiana University and at the Seventh Latin American Meeting of the Econometric Society held in Sao Paulo in August 1986. It was supported in part by the Bureau of Business and Economic Research at the University of Illinois and in part by an NSF grant. Both sources of support are gratefully acknowledged. 


\section{REFERENCES}

1. S. K. Berberian, Measure and integration, Chelsea, New York, 1965.

2. C. Berge, Topological spaces, Macmillan, New York, 1963.

3. P. Billingsley, Convergence of probability measures, Wiley, New York, 1968.

4. C. Castaing and M. Valadier, Convex analysis and measurable multifunctions, Lecture Notes in Math., vol. 580, Springer-Verlag, New York, 1977.

5. P. Dasgupta and E. Maskin, The existence of equilibrium in discontinuous economic games, Theory and Applications, Rev. of Econom. Stud. 53 (1986), 1-35.

6. S. Dolecki, G. Salinetti, and R. J. Wets, Convergence of functions: equi-semicontinuity, Trans. Amer. Math. Soc. 276 (1) (1983), 409-429.

7. J. Dugundji, Topology, Allyn \& Bacon, Boston, Mass., 1966.

8. G. A. Edgar, Disintegration of measures and the vector-valued Radon-Nikodym theorem, Duke Math. J. 42 (3) (1975), 447-450.

9. S. Hart, W. Hildenbrand, and E. Kohlberg, On equilibrium allocations as distributions on the commodity space, J. Math. Econom. 1 (1974), 159-167.

10. W. Hildenbrand, Core and equlibria of a large economy, Princeton University Press, Princeton, N.J., 1974.

11. J. Hoffman-Jorgenson, Probablity in B-spaces, Lecture Notes Series, vol. 48, Aarhus Universitet, Matematisk Institut, Aarhus, 1977.

12. E. Klein and A. C. Thompson, Theory of correspondences, Wiley, New York, 1984.

13. M. Ali Khan, Equilibrium points of nonatomic games over a Banach space, Trans. Amer. Math. Soc. 293 (1985), 737-749.

14. M. Ali Khan and N. Papageorgiou, On Cournot-Nash equilibria in generalized qualitative games with a continuum of players, Nonlinear Anal. 11 (1987), 741-755.

15. __ On Cournot-Nash equilibria in generalized qualitative games with an atomless measure space of agents, Proc. Amer. Math. Soc. 100 (1987), 505-510.

16. A. Mas-Colell, On a theorem of Schmeidler, J. Math. Econom. 13 (1984), 201-206.

17. K. Morita, Products of normal spaces with metric spaces, Math. Ann. 154 (4) (1964), 365-382.

18. J. Nagata, Modern general topology, North-Holland, Amsterdam, 1968.

19. K. Parthasarathy, Probability measures on metric spaces, Academic Press, New York, 1967.

20. G. Salinetti and R. Wets, On the hypo-convergence of probability measures, Optimization and Related Fields (R. Çonti et al., eds.), Lecture Notes in Math., vol. 1190, Springer-Verlag, New York, 1986.

21. D. Schmeidler, Equilibrium points on non-atomic games, J. Statist. Phys. 7 (1973), 295-300.

22. L. Schwartz, Radon measures on arbitrary topological spaces and cylindrical measures, Oxford University Press, London, 1973.

23. F. Topsoe, Topology and measure, Lecture Notes in Math., vol. 133, Springer-Verlag, New York, 1970.

24. R. J.-B. Wets, On a compactness theorem for epi-convergent sequences of functions, Mathematical Programming (R. W. Cottle et al., eds.), Elsevier Science Publishers, Amsterdam, 1984.

25. S. Willard, General topology, Addison-Wesley, New York, 1970.

Department of Economics, University of Illinois, Champaign, Illinois 61820

Current address: Department of Political Economy, The Johns Hopkins University, Baltimore, Maryland 21218 